\title{
ALMA IM ÖSTERREICHISCHEN BIBLIOTHEIKENVERBUND (OBV): AUS DER WERKSTATT DER OBVSG
}

\section{von Bettina Kann}

Zusammenfassung: Im März 2018 nahm die sogenannte Netzwerkzone als dritte Einheit nach den Bibliotheken der Kohorten 1 und 2 den Betrieb auf. Nach einer mehrjährigen Projektlaufzeit ist damit der neue Kern der Verbundarchitektur erfolgreich implementiert worden. Der Aufsatz behandelt einerseits die neue Verbundarchitektur mit dem Parallelbetrieb zwischen Alma und Aleph und die Umstellung der verschiedenen zentralen Services auf diese neue Architektur.

Schlüsselwörter: Alma; Aleph; Österreichischer Bibliothekenverbund

\section{ALMA IN THE AUSTRIAN LIBRARY NETWORK (OBV): FROM THE PERSPECTIVE OF THE AUSTRIAN LIBRARY NETWORK AND SERVICE LTD}

Abstract: In March 2018 the new Network Zone together with 13 Institution Zones linked went live with Alma as the nucleus of the new network architecture of the Austrian Library Network. Still more than 50 institutions in the Austrian consortium use Aleph as library system and will switch to Alma in the next years. One of the main goals in the project was a fully functional parallel operation between Aleph and Alma. The article will start explaining how the two systems are synchronized on bibliographic level (including MAB to MARC converter) and how inventory from member institutions in Aleph is displayed in Alma. It continues with an overview about additonal services that have to adapted to the two systems (e.g. workflow for reminders, Visual Library, GND, Primo and much more).

Keywords: Alma; Aleph; Austrian Library Network

DOI: https://doi.org/10.31263/voebm.v71i2.2133

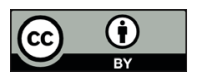




\section{Inhalt}

1. Projektorganisation

2. Projektphasen

3. Kreiskonvertierung, Kreishybridisierung und Katalogisierung

4. Parallelbetrieb

5. Zentrale Services

6. GND

7. Primo4Alma

8. Ein Blick auf Wave drei bis neun

\section{Projektorganisation}

Jene dreizehn Bibliotheken und die OBVSG, welche nach Alma umgestiegen sind, wurden in zwei Gruppen („Kohorten“1) plus Verbundumgebung eingeteilt. Jede der zwei Kohorten wurde von einem eigenen Kohortenmanager/einer Kohortenmanagerin koordiniert. Diese arbeiteten eng mit der Gesamtprojektkoordination, welche mit zwei Personen von der OBVSG übernommen wurde, zusammen. Zusätzlich stellte jede der beteiligten Einrichtungen eine/n lokalen Projektmanager/in, welche/r für die Koordination des Projekts innerhalb der eigenen Organisation zuständig war. Das Projektteam des OBV bestand somit aus 16 bis 18 Personen. Auf Seiten von ExLibris stand ebenfalls ein Projektteam für Organisation, Fachliches und Operatives zur Verfügung.

Neben umfangreichen organisatorischen Maßnahmen waren auch Expertinnen und Experten gefordert. Diese koordinierten sich in unterschiedlichen Gruppen, wobei diesen Gruppen von "Functional Experts", die jeweils für ein bestimmtes bibliothekarisches Fachgebiet zuständig waren, eine Schlüsselrolle zukam. So waren diese maßgeblich für umfangreiche Abnahmetests und Schulungen zuständig. Systembibliothekare, auf denen die Hauptlast der operativen Umstellung lag, Datenexperten und Entwickler, welche Funktionalitäten und Integrationen mit Alma-Mitteln, wie beispielsweise über APIs implementierten, ergänzten den Kreis der involvierten Personen.

Mit dem Abschluss des operativen Projekts Ende Mai 2018 endete auch die damit verbundene Organisationsstruktur. An ihre Stelle tritt das Wave-Management für die Migration aller nachfolgenden Waves, das zentral von der OBVSG durchgeführt wird. Die OBVSG wird darüber hinaus weiterhin, wo sinnvoll und notwendig, die Koordination zwischen ExLibris und den Verbundteilnehmern übernehmen und als zentraler Ansprechpartner für Fragen, die Netzwerkzone betreffend fungieren. 


\section{Projektphasen}

Das Projekt „Alma im OBV“ wurde mit dem Kick-off Meeting am 26. Jänner 2016 offiziell gestartet. Die Auftraggeber und ExLibris einigten sich auf einen Projektablauf, der im Wesentlichen aus drei Phasen bestand: Konzeptionsphase, Getting Ready Phase und Implementierung.

Als erste (Kohorte 1) gingen am 21. August 2017 folgende Bibliotheken mit dem neuen Bibliothekssystem in Betrieb:

- Bibliothek der FH St. Pölten

- Universitätsbibliothek der Technischen Universität Wien

- Universitätsbibliothek der Veterinärmedizinischen Universität Wien

- Universitätsbibliothek der Wirtschaftsuniversität Wien

- Universitätsbibliothek Wien

- Universitäts- und Landesbibliothek Tirol

Damit hatte auch der Parallelbetrieb von Aleph und Alma im OBV begonnen. Neben MAB-2 in Aleph wird nun MARC21 als Katalogisierungsformat in Alma verwendet. Der sogenannte MAB-MARC-Konverter, auf den später noch genauer eingegangen wird, sorgt dabei für die Datenkonvertierung zwischen den beiden Systemen.

Im Parallelbetrieb dieser ersten Phase wurden Bestandsnachweise der Alma Bibliotheken in Aleph nachgezogen. Dazu wurden auf der einen Seite die Bestandsinformationen aus Alma in regelmäßigen Intervallen exportiert und in Aleph importiert. Auf der anderen Seite wurden neue und geänderte bibliografische Datensätze in Aleph über den so genannten VSTServer in die einzelnen Alma Institutionszonen gespielt. Die Katalogisierung aus den Alma Institutionszonen wurde über den X-Server in Echtzeit mit der Verbunddatenbank ACC01 synchronisiert.

Die ersten Wochen gestalteten sich dabei turbulent, viele komplexe neue Workflows mussten erarbeitet, kommuniziert und erlernt werden. Auch die Synchronisierung der beiden Systeme erforderte bis zum Schluss Nachjustierungen.

Im Jänner 2018 gingen die Bibliotheken der Kohorte 2 mit Alma live:

- AK Bibliothek Wien für Sozialwissenschaften

- Österreichische Nationalbibliothek

- Universitätsbibliothek der Medizinischen Universität Wien

- Universitätsbibliothek Graz

- Universitätsbibliothek Linz

- Universitätsbibliothek Salzburg

- Verbund für Bildung und Kultur 
Während des Umstiegs der Bibliotheken fanden in der OBVSG bereits umfangreiche Vorarbeiten für die Migration der Verbunddatenbank ACC01 auf die Netzwerkzone statt. Letzte Datenkorrekturen wurden noch in den letzten Stunden vor dem Datenabzug durchgeführt. Am 13.März 2018 wurde die Alma Netzwerkzone offiziell in Betrieb genommen. Anschließend wurden bis inklusive 25. März alle Institutionszonen, die ja bis dahin als stand-alone Systeme von der Verbunddatenbank versorgt wurden, an die neue Netzwerkzone angebunden. Seit 13. März ist nun Alma das primäre System der neuen Verbundarchitektur. Das bisherige Aleph-Zentralsystem dient den Aleph-/Alephino-Bibliotheken bis zur Umstellung weiterhin als Katalogisierungssystem und wurde dazu mit der Netzwerkzone gekoppelt. Über diese sogenannte Aleph-Bridge werden die Daten der Aleph-/ Alephino-Bibliotheken in die Alma Netzwerkzone eingebracht. Bestandsnachweise der Aleph-/Alephino-Bibliotheken werden nun über die so genannten Bridge-Institutionen in die Netzwerkzone eingebracht. Aus dieser wird der Gesamtbestand des Österreichischen Bibliothekenverbundes über Primo zur Verfügung gestellt. Die kommenden Monate werden noch davon geprägt sein, Fehler zu beheben und einen reibungslos laufenden Betrieb zu gewährleisten. Dies bedeutet auch noch Nacharbeiten seitens ExLibris (Stichwort: GND). Beispiele für diese ersten Nachjustierungen waren die Wiedereinführung von Checks in Aleph, insbesondere der Dublettenkontrolle von AC-Nummern und ein Problem beim General Publishing der Daten aus Alma, das seit Go-Live der Kohorte 1 bestand.

\section{Kreiskonvertierung, Kreishybridisierung und Katalogisierung}

Der MAB-MARC Konverter wurde gemeinsam mit dem Experten bei ExLibris ständig weiter verbessert.

Seit Start der Kohorte 1 ist der MAB-MARC Konverter für die Datenkonversion der bibliografischen Daten zwischen Aleph und Alma (in beiden Richtungen) erfolgreich im Einsatz. Zusätzlich zur MAB-MARC Konvertierung ist auch die sogenannte „Kreishybridisierung“ produktiv, bei der RAK Datensätze beim Rückimport nach Aleph auf RDA gehoben werden.

Folgende weitere Maßnahmen wurden durchgeführt, um soweit als möglich, Hilfestellung bei der Katalogisierung zu geben, um eine einheitliche Vorgangsweise im Verbund sicher zu stellen.

- Erstellung und Pflege (gemeinsam mit den Functional Experts für Resource Management) einer ASEQ-MARC21 Konkordanz für die Bearbeiter/innen. 
- Implementierung neuer und Optimierung bestehender Normalisierungsregeln in Alma für

- Abspeichern von Datensätzen

- Import und Importprofile für externe Ressourcen, eBook-Datenpakete, Visual Library...

* Satz erweitern (Ersatz für „Satz ableiten“)

- Merge-Routinen für Anreicherung aus externen Ressourcen

- Erstellung und Pflege der zentralen Katalogisierungsschablonen

- Pflege des MARC21-Extension-Packs inkl. Controlled Vocabulary

- Anleitungen für Bearbeiter/innen und Systembibliothekar/innen im externen Wiki der OBVSG zu Themenbereichen wie Zusammenführen von Datensätzen, Match-Routinen, Verlinken von Datensätzen mit der Netzwerkzone u.v.m.

Mit Produktivnahme der Netzwerkzone im März 2018 können die Katalogisierungsschablonen geteilt werden und sind somit bei Aktualisierung in der Netzwerkzone auch sofort in der jeweiligen Institutionszone verfügbar.

Ein neu eingeführter Bereich im OBVSG-Ticketsystem für Rückmeldungen zur Katalogisierung in Alma (sowohl vor als auch nach Produktivnahme der Netzwerkzone) ist die Basis für Korrekturen und Optimierungen des MAB-MARC Konverters als auch für Korrekturen und Optimierungen von Normalisierungsregeln und weiteren Set-Up Einstellungen im Bereich der bibliografischen Daten in Alma.

\section{Parallelbetrieb}

Einer der zentralen Punkte in der Auftragsvergabe war die Erfordernis eines funktionierenden parallelen Betriebs von Alma und Aleph. Von Beginn an war klar, dass die Umstellung auf das neue Bibliothekssystem mehrere Jahre in Anspruch nehmen wird und auch Aleph Bibliotheken weiterhin ohne Reibungsverluste im gewohnten System arbeiten müssen. Nach dem Umstieg der Kohorte 1 auf Alma trat der Parallelbetrieb Phase 1 in Kraft, während dessen Aleph noch das führende System war. Alle Änderungen an bibliographischen Datensätzen sowie Löschungen und neue Sätze wurden über den so genannten VST-Server direkt in die einzelnen Alma Institutionszonen gespielt. Die Zuweisung eines Datensatzes zu einer Institutionszone erfolgte direkt im bibliografischen Datensatz mit dem LOW-Feld. War zB im einem Datensatz ein LOW \$\$aA-UBI vorhan- 
den, so wurde der Datensatz an die Institutionszone der Universitätsbibliothek Innsbruck geschickt. Im Gegensatz dazu wurden die Holdings und Items der Alma Bibliotheken aus den einzelnen Institutionszonen exportiert und in die ACC60 bzw. z300 importiert. Auf diese Weise war gewährleistet, dass der zentrale Aleph Katalog die Informationen aller Verbundteilnehmer verfügbar hatte. Auch das Publishing für die zentrale Primo View erfolgte noch aus der ACC01. Mit dem Umstieg der Verbundzentrale auf die Netzwerkzone wurde Letztere zum führenden System, das ab dem 13.3.2018 auch alleine die Vergabe der AC-Nummer steuert. Aleph wurde zum nachgeordneten System, welches die AC-Nummer nun von der Netzwerkzone bezieht. Für den bibliografischen Datenaustausch ist weiterhin der in Aleph angesiedelte MAB-MARC Konverter das Herzstück.

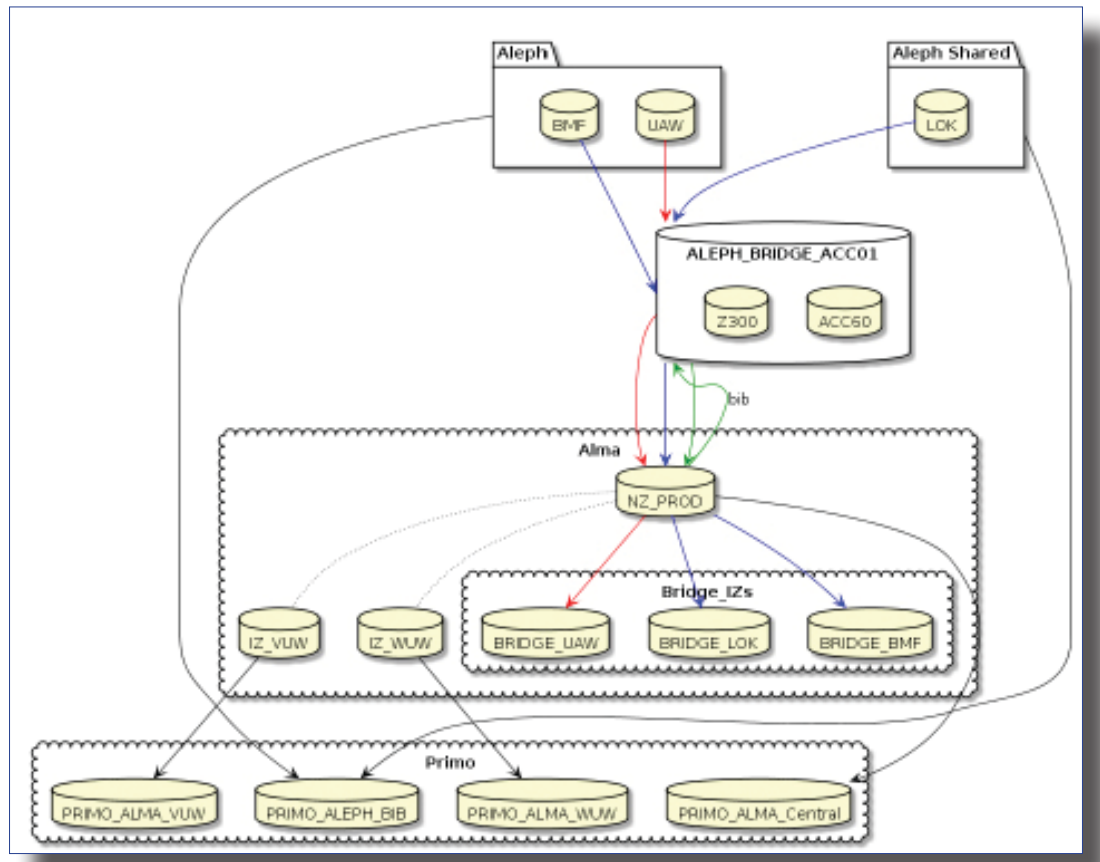

Abb. 1: Parallelbetrieb Phase 2

Für die Bearbeiterinnen und Bearbeiter hat sich der Workflow in Aleph nur geringfügig geändert, Datensätze werden sofort beim Abspeichern des Datensatzes in die Netzwerkzone und zurück geschickt. Datensätze, welche von Alma-Bibliotheken direkt in der Netzwerkzone geändert bzw. neu 
angelegt werden, werden stündlich aus der Netzwerkzone exportiert und in die ACC01 geladen.

Das Inventar der Aleph-Bibliotheken wird einmal täglich aus Aleph exportiert und über ein so genanntes Integrationsprofil in die 54 BridgeInstitutionszonen aufgeteilt. Diese sind wiederum mit der Netzwerkzone verlinkt, sodass deren Holdings und Items auch in der Netzwerkzone sichtbar sind und ebenso wie das Inventar der Alma Bibliotheken in die zentrale Primo View exportiert werden. Eine spezielle Bridge-Institution beinhaltet das Inventar der Nicht-Aleph-Bibliotheken bzw. alte ÖZDB-Daten u.ä. (Abbildung 1 verdeutlicht die Architektur eingehender).

\section{Zentrale Services}

\section{OPUS}

Wie geplant wurde die Dissertationsdatenbank „OPUS“ Anfang 2018 eingestellt und die Daten in andere Systeme übergeführt. Letzte Korrekturen und Änderungen konnten von Institutionen während einer eingerichteten Übergangsphase noch vorgenommen werden.

\section{Upgrade2AC}

Der bisher in Aleph laufende Service „Upgrade2AC“, welcher es ermöglicht, lokale Datenbestände automatisiert in die zentrale Verbunddatenbank ACC01 zu heben, wurde mit dem Alma-Job „Link Set of Records with Networkzone" ersetzt. Für Aleph-Bibliotheken funktionert der Upgrade2AC Prozess wie bisher.

\section{SAP}

Plangemäß wurde die SAP-Anbindung von Alma mit dem Go-Live der Kohorte 1 im August 2017 realisiert. Dabei werden die Rechnungsdaten aus Alma in einem definierten XML-Format exportiert, über eine eigens entwickelte Schnittstelle nach SAP geschickt und dort weiterverarbeitet. Die von SAP erzeugte Rückmeldung über den Status der Rechnung wird wiederum in das für Alma geeignete Format umgewandelt und automatisch nach Alma importiert. Damit erhält die Rechnung im System selbst den aktuellen Status (z.B. „Paid“). 
Mit Ende April konnte auch der neue eDOC Dienst seinen Vollbetrieb aufnehmen. Die Ablöse der alten Swish-E Suchoberfläche durch den eDOC-Objekte-Datenmonitor wurde bereits im März 2018 durchgeführt.

\section{Visual Library}

Die Adaptionen für den Dienst Visual Library gestalteten sich umfangreicher als ursprünglich angenommen, dieser Dienst wurde daher erst im Juni 2018 wiederaufgenommen. Aus der Visual Library Applikation werden die Datensätze wie bisher im Format MAB exportiert. Visual Library und Alma/Aleph arbeiten weiterhin mit der AC-Nummer als verbindendem Identifier. Die erste umfangreiche Umstellung bestand bereits darin, dass Alma im Gegensatz zu Aleph nicht mehr mit angelieferten AC-Nummern bei neuen Datensätzen arbeiten kann, sondern die AC-Nummer in Alma direkt erzeugt werden muss. Daher muss sich Visual Library für jeden neuen Datensatz über die Alma-API durch Anlegen eines Dummy-Datensatzes eine AC-Nummer holen und dem bibliographischen Datensatz in VL zuweisen. Die dann von Visual Library exportierten Datensätze werden nachdem der URN registriert wurde nach Marc21 konvertiert und in die Alma Netzwerkzone geladen. Danach werden sie einerseits den Institutionen über FTP zum Import in deren Institutionszonen zur Verfügung gestellt, anderseits gehen sie über die „normale“ Alma-Aleph Synchronisierung von der Netzwerkzone in die ACC01. In einem ebenfalls mit Alma neu aufgesetzten Prozess, werden die Aleph Lokalsysteme versorgt (push). Im letzten Schritt werden die bibliographischen Datensätze an Visual Library zurück geliefert. Die digitalen Dokumente selbst werden über einen modifizierten eDOC Workflow volltextindexiert und sind im Anschluss über Primo auffindbar.

Noch für 2018 ist der Start der Umstellung des Datenexports aus VL im Format MARC21 geplant, mit der die Neuentwicklung und damit Vereinfachung des Workflows beabsichtigt ist.

\section{Memo-Workflow}

Völlig auf neue Beine gestellt werden, müssen die bisherigen Memo-Workflows, welche nun unter anderem Prüfroutinen, Modifikationen und Löschungen in beiden Systemen (Alma und Aleph) berücksichtigen. Die neuen Prüfroutinen für Löschmemos in Alma umfassen beispielsweise: 
- Prüfungen auf weitere Memos am gleichen Datensatz

- auf eventuell noch vorhandene Holdings und Items in Alma und/ oder Aleph

- auf Verlinkungen zu und von anderen bibliographischen Datensätzen

- aufVorhandensein von eDOC und/oder VL-Dokumente.

\section{IV-Scans und Links zu digitalisierten Werken aus dem „ABO“-Projekt der Österreichischen Nationalbibliothek}

Diese wurden in den letzten Monaten ebenfalls nach Alma umgestellt. Die bereits erwähnte unterschiedliche Funktionsweise von Alma brachte es mit sich, dass die Datensätze nun von der ÖNB selbst angereichert und in die Netzwerkzone eingebracht werden. Die Weiterverarbeitung der pdfDateien aus IV-Scan über den eDOC-Workflow und über das adaptierte PPS nach Primo wird weiterhin von der OBVSG durchgeführt.

\section{eBook}

Der eBook-Dienst wurde ebenfalls einem Relaunch unterzogen und konnte im April 2018 wieder aufgenommen werden. Gerade beim E-Book Dienst, aber auch beim neuen Workflow für Visual Library hat sich gezeigt, dass die Alma Architektur hinsichtlich der "Replikation“ konträr zu Aleph ist und daher die in Aleph rein zentral durchgeführten Schritte jetzt zusätzlichen Handlungsbedarf in den einzelnen Institutionen bedingen. In Aleph wurden die Datensätze direkt in die ACC01 gespielt und danach in die betroffenen Lokalsysteme von oben nach unten gepusht. In Alma werden sie nun in die Netzwerkzone gespielt, exportiert und den betroffenen Institutionen auf einem FTP-Server zur Verfügung gestellt. Von dort können sie dann von den einzelnen Institutionen in deren Institutionszonen importiert und mit der Netzwerkzone verlinkt werden. Die im Mai neu eingerichtete Expertengruppe E-Medien wird in den nächsten Monaten die Workflows für die verschiedenen Mediengruppen weiter ausbauen.

Basis für die Integration aller Dienste in Alma ist unter anderem die Alma-API, die einen Zugriff auf Daten, Jobs, Reports und Konfigurationen ermöglicht. Um die Möglichkeiten dieser Schnittstelle flexibel auch in bestehenden Programmen nutzen zu können, wurde als erster Schritt ein API-Framework für Perl entwickelt. Diese Programmbibliothek ermöglicht Alma-Funktionalitäten direkt von zentralen Diensten aus zu steuern und Daten auszutauschen. 


\section{GND}

Die in Alma bereits vorhandenen Normdateien (LC Names, LCSH, BARE etc.) sind deutlich anders strukturiert als die GND, weshalb im Bereich der Normdatennutzung zahlreiche Adaptionen notwendig waren (bspw. Verlinkung über Identifier, anstatt über Textstrings; häufige Updates und die damit einhergehenden Korrekturen in den Titeldaten). Was die aktive Normdatenarbeit (Erstellen und Editieren von GND-Datensätzen), welche in der GND kooperativ von tausenden Kolleginnen und Kollegen betrieben wird, betrifft, mussten in Alma zahlreiche Funktionalitäten erst gänzlich neu geschaffen werden.

Prinzipiell haben mit Alma in Bezug auf die GND in dem einen oder anderen Bereich deutliche Verbesserungen Einzug gehalten (bspw. Verringerung des Update-Intervalls von einer Woche auf sechs Stunden), insgesamt muss jedoch leider bemerkt werden, dass während die Normdatennutzung (F3Funktionalität) mittlerweile annähernd fehlerfrei funktioniert, dies für die im Hintergrund ablaufenden Korrekturen der Titeldaten aufgrund von Updates der GND-Daten (Änderungen der bevorzugten oder abweichenden Benennungen, Löschungen, Umlenkungen, Teilumlenkungen etc.) nicht der Fall ist.

Der Start der produktiven Phase der redaktionellen GND-Arbeit (Neueingabe von GND-Datensätze, Ergänzungen/Korrekturen an bestehenden Datensätzen etc.) musste bereits mehrmals verschoben werden. Verantwortlich hierfür ist, neben einer Änderung im GND-Datenformat im Herbst 2017, auf die weder Ex Libris noch der OBV Einfluss hatten, die Tatsache, dass die Online-Kommunikation zwischen Alma und der GND-Quelldatei an der DNB, trotz langwieriger vorbereitender Arbeiten und monatelanger Bemühungen um Behebung der Probleme, noch immer nicht zufriedenstellend läuft. Wenngleich wir seitens des OBV hoffen diesbezüglich im Herbst von Aleph auf Alma umsteigen zu können, lässt sich zum jetzigen Zeitpunkt nicht seriös abschätzen, wann Ex Libris die von uns gemeldeten Mängel beheben wird.

\section{Primo4Alma}

Mit der Umstellung auf Alma änderte sich auch die Datenversorgung dieser Bibliotheken. Seit Go-Live kommen die Daten direkt aus dem AlmaPublishing, welches viermal am Tag alle sechs Stunden läuft. Ab 23:00 Uhr holen sich die Pipes die Daten und laden diese in die jeweilige Primo-View der einzelnen Institutionen. 
Zusätzlich zu diesen Daten wurden auch die bereits vorhandenen IVScans und E-Books nachgeladen. Hierzu wurden die Daten aus dem alten System exportiert und mittels des MAB2MARC-Konverters konvertiert und nach Primo geladen. Auch die bereits vorhandenen eDOC Daten wurden in das neue System zeitgerecht überspielt und in die Volltextindexierung inkludiert.

Der Umstieg auf das neue Primo4Alma gestaltete sich für die Bibliotheken der Kohorte 2 leichter, da sie bereits auf Vorhandenes aufbauen konnten. So gab es zum Beispiel schon ein brauchbares Standard-Normalisierungs-Template, von dem die Bibliotheken dann ihre spezifische Normalisierung ableiten konnten.

Neu hinzu kam ein neues Testsystem Primo4Alma. Da Tests nicht auf der produktiven Primo4Alma Instanz durchführen wurden, da sonst der Betrieb der Kohorte 1 gestört gewesen wäre, wurde das Primo4Alma-Produkionssystem kopiert und ein Primo4Alma-Testsystem davon abgeleitet. Auf diesem System konnten die Bibliotheken der Kohorte 2 ihre Tests im Bereich Primo durchführen.

Bei den Überlegungen zur neuen Verbundsuchmaschine, wurde relativ früh entschieden, dass die OBVSG nicht auf das neue Primo4Alma-System wechselt, sondern dass von der bereits vorhandenen Primo4Alma Instanz ein Klon erstellt wird. Damit konnten Unterbrechungen für die anderen Bibliotheken beim Go Live bzw. beim Laden der Daten vermieden werden.

Dies hatte auch die Änderung des View-Code von ACC auf OBV zur Folge. Speziell im Bereich der Bestandsangabe im Standorte-Tab gab es einige Herausforderungen. Da die Non-Alma-Bibliotheken ebenfalls in Primo angezeigt werden, wurden diese Bibliotheken als „Bridge-Institutionen" in Alma angelegt. Diese Architektur gewährleistet, dass alle Bestände gepublisht werden, egal in welchem Bibliothekssystem sie erfasst sind. Die Bestandsanzeige für diese „Bridge-Institutionen“ bzw. das Durchlinken auf die Primo Views dieser Bibliotheken musste im Details analysiert und bis auf Sublibrary-Ebene konfiguriert und gemappt werden.

Eine weitere Herausforderung war die Umstellung von PPS. Im Gegensatz zum vorigen Aleph-PPS, muss es jetzt nur mehr die Daten für die OBV-View vorbereiten. Zusätzlich sollen jedoch auch die IV-Scans \& eBooks für die Bibliotheken bereitgestellt werden. Dazu wurden verschiedenste Abfragen mittels SRU und API eingebaut. Die anderen Alma-Primo-Bibliotheken werden nun über eine andere Datenversorgung beliefert. Diese Datenquelle wird nun mittels einer eigenen Datasource und Pipe den Bibliotheken zur Verfügung gestellt. Das gleiche Prozedere wird auch für eBooks verwendet. 
Mit Mitte April wurde die Verbundsuchmaschine umgestellt. Ziel war, alle Funktionalität der alten ACC-View in die neue OBV-View zu übernehmen. Eine größere Umstellung gab es im Bereich der „Nachlässe und Handschriften". Diese Daten kamen in Aleph aus einer eigenen Datenquelle (ACC05) und wurden getrennt vom anderen Bestand normalisiert. Das hat sich mit der Umstellung auf Alma geändert, nun kommen die Daten aus der gleichen Datenquelle und laufen über die gleiche Normalisierung.

\section{Ein Blick auf Wave drei bis neun}

Nach Projektabschluss ist vor Projektbeginn - so könnte das Motto lauten, denn: wohl ist die Umstellung für Kohorte 1, 2 und OBVSG nun beendet, für alle noch umsteigenden Bibliotheken beginnt die Arbeit aber erst bzw. hat sie eben begonnen. Mit dem Kick-Off am 19. März 2018 hat der operative Umstieg der Wave 3 begonnen. Wie eingangs erwähnt hat sich damit auch die Organisationsstruktur geändert. Zwar stellt jede migrierende Einrichtung weiterhin eine/m Projektmanager/in, das Wavemanagement wird jedoch von der OBVSG zentral übernommen. Weiters sind die zeitlichen Vorgaben für die Institutionen ab Wave 3 wesentlich enger, die Umstellung einer Wave dauert zukünftig ca. ein halbes Jahr. FH Burgenland (Alephino), Universität Klagenfurt und Universität für Bodenkultur haben bereits erfolgreich die Migration durchgeführt und sind Anfang September mit Alma live gegangen.

Auch die Wave 4 hat sich bereits konsolidiert, diese Bibliotheken haben am 4. Juli ihr „onboarding-meeting“ (= Kick-off) und werden bereits im Jänner 2019 live gehen. Wave 4 besteht bereits bis auf zwei Bibliotheken ausschließlich aus so genannten „Sharing-Einrichtungen“, dh. die Bibliotheken teilen sich eine BIB-library in Aleph, haben aber jeweils eigene ADMs zugewiesen. Diese Einrichtungen werden systembibliothekarisch ebenfalls von der OBVSG betreut. Das Konzept „Sharing“ selbst wird nach intensiven Diskussionen aufgegeben, jede Einrichtung wird in Alma eine eigene individuelle Institutionszone erhalten. Die Vorteile liegen auf der Hand: Homogenität der Architektur im gesamten Verbund bei gleichzeitiger Individualisierbarkeit der Konfiguration für die einzelnen Einrichtungen. Der ausschlaggebende Punkt war jedoch, dass anders nicht zu hundert Prozent hätte gewährleistet werden können, dass Finanz und Userdaten komplett gegeneinander abzuschotten gewesen wären. Das gewählte Modell einzelner Institutionszonen stellt dies sicher. 
2021 werden dann mit Wave 9 die letzten Bibliotheken nach Alma migriert sein. Die komplette Umstellung des Österreichischen Bibliothekenverbundes ist damit abgeschlossen und die Planungen für die nächsten Großprojekte können beginnen.

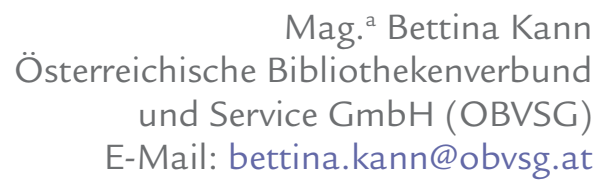

1 „Kohorte“ wurde als Begriff für eine Gruppe zeitgleich in Alma implementierter Institutionen von „Wave“ abgelöst. 